\title{
Escitalopram-induced extrapyramidal symptoms
}

\begin{abstract}
Serotonin specific reuptake inhibitors (SSRI) are the commonly used antidepressants for a variety of clinical conditions and have gained wide popularity. They are sometimes associated with extrapyramidal side effects (EPS) including Parkinsonism. The induction of EPS by SSRIs has been thought to be a consequence of serotonergically mediated inhibition of the dopaminergic system. We would like to present a case of escitalopram-induced Parkinsonism to increase awareness about this clinical problem. Prompt discontinuation of the offending drug resulted in complete relief of symptoms.
\end{abstract}

Keywords: escitalopram, extrapyramidal symptoms, parkinsonism

\author{
Volume 8 Issue 3 - 2018
}

\author{
Suresh Kumar PN,' Arun Gopalakrishnan² \\ 'Department of Psychiatry, KMCT Medical College, India \\ ${ }^{2}$ Consultant Psychiatrist, PVS Hospital, India
}

Correspondence: Suresh Kumar PN, Anaswara,Vazhathuruthi Road, P.O.Civil Station, Kozhikode-673020, Calicut, Kerala, Tel -0495-2372684, 94472 I8825, Email drpnsuresh@gmail.com

Received: December 19, 2017| Published: May 22, 2018

\section{Introduction}

Serotonin specific reuptake inhibitors (SSRI) are widely used antidepressants for a variety of clinical conditions because of their relatively safe side-effect profile. However, a recent review of all Food and Drug Administration adverse events indicates that SSRIs are common offenders among the various classes of antidepressants, producing extrapyramidal symptoms (EPS). The incidence of EPS is high with escitalopram (12\%) followed by sertaline $(11 \%)$, paroxetine $(10 \%)$ and fluoxeine $(8 \%) .{ }^{1}$ The SSRIs produces reversible or irreversible motor disturbances through pathophysiological changes in basal ganglion motor system by altering the dopamine receptors postsynaptically. ${ }^{2}$ Here we report a case of escitalopram-induced EPS to increase clinical awareness about this rare presentation.

\section{Case report}

A 34-year-old female was presented to the psychiatry outpatient department complaining of sadness, weeping spells, loneliness, decreased interest in day to day activities, lethargy and disturbed biological functions over the past six months. Her past, family history and personal history were non-contributory. General and systemic examination was within normal limits. Mental status examination revealed a young female with decreased psychomotor activity, depressed affect, depressive cognitions with well preserved insight. A diagnosis of moderate depressive disorder was made and she was started on escitalopram $10 \mathrm{mg}$ per day along with clonazepam $0.5 \mathrm{mg}$ at night. She was faring well and escitalopram $10 \mathrm{mg}$ was continued for next 18 months as per patient's request. In the $18^{\text {th }}$ month, she was presented in the OPD with complaints of slurring of speech, drooling of saliva, decreased bodily movements and slowness in walking. Neurological examination revealed bradykinesia, cogwheel rigidity, dysarthric speech and tremor of hands. Routine biochemical tests and head MRI and EEG were done and found to be normal. No history of any other drug use/abuse, head injury, seizures, fever or movement disorder was reported. A diagnosis of escitalopram-induced acute extrapyramidal syndrome was made after excluding other possible causes like infections, stroke, poisoning, seizures, familial movement disorders or structural brain abnormalities. Escitalopram was immediately discontinued, patient was reassured, and trihexyphenidyl $2 \mathrm{mg}$ bid was started. Follow-up after 2 weeks reported significant improvement in symptoms. Subsequently trihexyphenidyl was stopped and dothiapin 50mg per day was started to control the depressive symptoms. The patient was followed-up for next 6 months without any symptom recurrence. The Naranjo probability scale applied retrospectively revealed a score of 6 , pointing to a "probable" drug reaction.

\section{Discussion}

The data for escitalopram induced EPS is rare except in few cases reported previously. ${ }^{3-5}$ Though it is a rare side effect of escitalopram, it is one the clinician must be aware of and be ready to handle. The potential risk factors for EPS induced by antidepressants are advancing age, female gender, pre-existing extrapyramidal disorder, other concurrent neuroleptic medications and pharmacokinetic interaction through CYP2D6 inhibition. ${ }^{1}$ Other than the female gender none of these risk factors were present in our patient. Researchers have extrapolated a link between antidepressant induced EPS and the CYP2D6 phenotype. On analysing the risk factors for these reactions during treatment with SSRIs, including the CYP2D6 phenotype, serotonin and dopamine transporter and receptor polymorphisms, it was noted that the risk of EPS with SSRIs seemed to increase with advanced age and the presence of the A1 allele of the dopamine D2 receptor (DRD2) gene Taq1A. ${ }^{6}$

Being a young patient the possibility of young onset parkinsonian disease (YOPD) should also be considered in the index case. In YOPD, family history of parkinsonism is an important risk factor because of genetic involvement. ${ }^{7}$ Our patient's history was not significant for either parkinsonism or any other movement disorder. In rat models of EPS induced by SSRIs, a specific effect on tyrosine hydroxylase, which is a speed-limiting enzyme of dopamine synthesis in substantia nigra has been reported. Tyrosine hydroxylase synthesis was significantly decreased by SSRIs in substantia nigra, and the serotonin transporter (SERT) inhibition can activate microglia and alter the regulation of tyrosine hydroxylase, the rate limiting enzyme for dopamine biosynthesis, and these changes may play a role in mediating the EPS associated with SERT inhibitors. ${ }^{8}$

It has been reported that drug induced parkinsonism (DIP) may represent a pre-existing vulnerability to future Parkinson's disease. ${ }^{9}$ Treatment of DIP involves discontinuation of the offending drugs, which usually promotes remission of the parkinsonian syndrome within a short time, although parkinsonism may sometimes persist 
and require dopaminergic treatment as it happened in cases elsewhere. DIP is clinically indistinguishable from Parkinson's disease. Singlephoton emission computed tomography (SPECT) imaging may help to determine whether DIP is entirely drug-induced or an exacerbation of subclinical PD. ${ }^{10}$

Our patient was relatively young, female, fulfilled the clinical diagnostic criteria for drug induced EPS i.e the presence of parkinsonism, no history of parkinsonism before the use of the SSRI, and onset of parkinsonian symptoms during use of the offending drug. One atypical feature was that compared to previously reported cases where EPS developed within a few weeks after initiation of escitalopram, in the index patient, EPS developed only two years after starting escitalopram. However, the EPS was reversed after a short course of anticholinergic treatment. The reason for late onset of EPS needs further exploration. There was no known concomitant offending drug used beside SSRI.

\section{Conclusion}

Escitalopram is a widely used antidepressant in a variety of psychiatric disorders. Clinicians should be careful if any motor symptoms appear in patients using escitalopram even after months or years of therapy as it can lead to diagnostic or treatment errors. This case report was an attempt to create awareness about drug induced EPS as adverse reaction in patients taking escitalopram so that timely recognition can prevent such adverse effects.

\section{Acknowledgements}

None.

\section{Conflict of interest}

The authors declares no conflict of interest.

\section{Patient consent form}

The patient provided written informed consent and gave the permission for this case report.

\section{References}

1. Madhusoodanan S, Alexeenko L, Sanders R, et al. Extrapyramidal symptoms associated with antidepressants-a review of the literature and an analysis of spontaneous reports. Ann Clin Psychiatry. 2010;22(3):148156.

2. Lane RM. SSRI-induced extrapyramidal side-effects and akathisia: implications for treatment. J Psychopharmacol. 1998;12(2):192-214.

3. Dixit S, Khan SA, Azad. A case of SSRI induced irreversible parkinsonism. J Clin Diagno Resear. 2015;9(2):VD01-VD02.

4. Nimber JS, Aggarwal A. Acute extrapyramidal syndrome induced by escitalopram: a case report. Psychopharmacol. 2014;231(18):3813-3814.

5. Dave-Momin N, Lokhande C, Shah N, et al. Escitalopram induced dystonia: a case report. Int J Toxicol Pharmacol Res. 2014;6:20-21.

6. Hedenmalm K, Güzey C, Dahl ML, et al. Risk factors for extrapyramidal symptoms during treatment with selective serotonin reuptake inhibitors, including cytochrome P-450 enzyme, and serotonin a d dopamine transporter and receptor polymorphisms. J Clin Psychopharmacol. 2006;22(6):192-197.

7. Schrag A, Ben-Shlomo Y, Brown R, et al. Young-onset Parkinson's disease revisited-clinical features, natural history, and mortality. Mov Disord. 1998;13(6):885-894.

8. MacGillivray L, Reynolds KB, Sickand M, et al. Inhibition of the serotonin transporter induces microglial activation and downregulation of dopaminergic neurons in the substantia nigra. Synapse. 2011;65(11):11661172 .

9. Gonul A, Aksu M. SSRI-induced Parkinsonism may be an early sign of future Parkinson's Disease. J Clin Psychiatry. 1999;60(6):410.

10. Lorberboym M, Treves T, Melamed E, et al. FP/CIT SPECT imaging for distinguishing drug-induced parkinsonism from Parkinson's disease. Mov Disord. 2006;21(4):510-514. 